\title{
A Finite Difference Equivalent Circuit Approach to Secondary Current Modelling in Annular Porous Electrodes
}

T. W. FARRELL

t.farrell@qut.edu.au

Centre in Statistical Science and Industrial Mathematics, Queensland University of Technology, GPO Box 2434, Brisbane Q 4001, Australia

D. L. S. MCELWAIN

s.mcelwain@qut.edu.au

Centre in Statistical Science and Industrial Mathematics, Queensland University of Technology, GPO Box 2434, Brisbane Q 4001, Australia

D. A. J. SWINKELS

doms@paracelsus.newcastle.edu.au Department of Chemistry, The University of Newcastle, Callaghan NSW 2308, Australia

\begin{abstract}
An equivalent circuit for an annular porous electrode is derived by reinterpreting the differential equations that approximate the distribution of voltage and current in such electrodes. The equivalent circuit is shown to provide useful physical interpretations of the secondary current distributions. Multi-loop circuit techniques are employed to obtain the current distributions within the circuit. The solutions are shown to compare well with the exact solutions of the model equations. In addition, the equivalent circuit approach is used to investigate the effect of curvature on the degree of polarization of the electrode.
\end{abstract}

Keywords: battery, equivalent circuit, finite differences, polarization, porous electrode

\section{Introduction}

Secondary current distribution (SCD) modelling provides a deeper understanding of the processes that determine the initial distribution of current in porous battery electrodes. The initial current distribution can set the trend for the discharge profile of the porous electrode at later times.

The analysis of secondary current in porous electrodes has been presented by Newman and Tiedemann [1] for rectilinear geometry and by Marshall [2] and Mak et al. [3] for cylindrical geometry. These authors develop a mathematical model based on the assumption of linear charge transfer kinetics and obtain exact solutions to the differential equations which approximate 
either the SCD or the potential distribution.

In the present paper we describe an alternative approach to obtaining the SCD for annular porous electrodes. This approach affords a good physical interpretation of the coupled system of differential equations which approximate the SCD in such electrodes. A physical interpretation of the equations is vitally important if a true understanding of system behavior is to be achieved. In addition, however, this approach to SCD modelling provides a physical model which may be directly compared to an annular porous electrode. The physical model developed here consists of an electric circuit comprised of a system of resistances. Like the governing equations of a mathematical model, the circuit represents the physical and electrochemical characteristics of various phases within the electrode. By determining the distribution of current within the circuit we obtain the SCD for an annular porous electrode. This method provides a relatively simple approach for SCD determination.

\section{Derivation of the Equivalent Circuit}

A general porous electrode of annular geometry is considered. The electrode may consist of a conductive electroactive material or a mixture of a semi-conducting electroactive material and an inactive conductor. Electrolyte solution is assumed to fill all voids within the porous electrode.

The basic equations which describe the conduction mechanism, the transfer of charge, the conservation of charge and the linearized activation polarization in an annular porous electrode are described in reference [3]. Combining these equations leads to a coupled system of differential equations which describe the surface overpotential, $\eta(r)(\mathrm{V})$, and the solution phase current, $i_{2}(r)(\mathrm{A})$, within the electrode, namely

$$
\frac{d \eta}{d r}=\frac{i_{2}}{A(r)}\left\{\frac{1}{\sigma}+\frac{1}{\kappa}\right\}-\frac{I}{2 \pi r H \sigma}
$$

and

$$
\frac{1}{A(r)} \frac{d i_{2}}{d r}=\frac{a i_{0}\left(\alpha_{a}+\alpha_{c}\right) F \eta}{R T} .
$$

Here $r(\mathrm{~cm})$ is the radial co-ordinate, $A(r)\left(\mathrm{cm}^{2}\right)$ is the cross-sectional area of the porous electrode in the radial direction, $\sigma$ and $\kappa(\mathrm{S} / \mathrm{cm})$ are the effective solid and solution phase conductivities respectively, $I(\mathrm{~A})$ is the total applied current, $H(\mathrm{~cm})$ is the height of the electrode, $a(/ \mathrm{cm})$ is 
the electrochemically active surface area of the pore walls per unit volume of the electrode, $i_{0}\left(\mathrm{~A} / \mathrm{cm}^{2}\right)$ is the exchange current density, $\alpha_{a}$ and $\alpha_{c}$ are the anodic and cathodic transfer coefficients, respectively, $F(\mathrm{C} / \mathrm{mol})$ is Faraday's Constant, $R(\mathrm{~J} / \mathrm{mol} \cdot \mathrm{K})$ is the gas constant and $T(\mathrm{~K})$ is the temperature.

Equations (1) and (2) may be discretized by rewriting the derivatives in terms of appropriate second order finite differences. Noting that $i_{1}(r)+$ $i_{2}(r)=I$, this gives

$$
\frac{\eta^{k+1}-\eta^{k-1}}{2 h}=\frac{i_{2}^{k}}{A^{k} \kappa}-\frac{i_{1}^{k}}{A^{k} \sigma}
$$

and

$$
\frac{i_{2}^{k+2}-i_{2}^{k}}{2 h A^{k+1}}=\frac{a i_{0}\left(\alpha_{a}+\alpha_{c}\right) F \eta^{k+1}}{R T}
$$

where $i_{1}(r)(\mathrm{A})$ is the solid phase current, $h(\mathrm{~cm})$ represents the distance between adjacent node points and $A^{k}=A\left(r^{k}\right)$. While both equations (3) and (4) are central difference approximations, the overpotential and the solid and solution phase currents are determined at node points which are offset from one another by the distance $h$.

In order to physically interpret equations (3) and (4) we consider Figure 1 which shows a section of circuit that has been overlaid onto a finite region of porous electrode. The circuit wires correspond to the current paths in the electrode. As shown in the figure, we denote the potential at the junctions in the circuit by $\Phi_{1}^{k-1}, \Phi_{2}^{k-1}, \Phi_{3}^{k+1}$ and $\Phi_{4}^{k+1}(\mathrm{~V})$.

If we let the width of the circuit section correspond to the distance $\Delta r$, between $N$ equally spaced node points in the porous electrode, where $\Delta r=$ $2 h(\mathrm{~cm})$, then the number of circuit sections in each electrode is $n$, where $n=N-1$. Equation (3) may be rewritten as

$$
\left(\Phi_{3}^{k+1}-\Phi_{4}^{k+1}\right)-\left(\Phi_{1}^{k-1}-\Phi_{2}^{k-1}\right)=i_{2}^{k} \frac{\Delta r}{A^{k} \kappa}-i_{1}^{k} \frac{\Delta r}{A^{k} \sigma} \quad(k=2,4, \ldots, 2 n)
$$

so that

$$
\left(\Phi_{2}^{k-1}-\Phi_{4}^{k+1}\right)-\left(\Phi_{1}^{k-1}-\Phi_{3}^{k+1}\right)=i_{2}^{k} \frac{\Delta r}{A^{k} \kappa}-i_{1}^{k} \frac{\Delta r}{A^{k} \sigma}
$$

which simplifies to

$$
V_{2}-V_{1}=i_{2}^{k} R_{2}^{k}-i_{1}^{k} R_{1}^{k}
$$




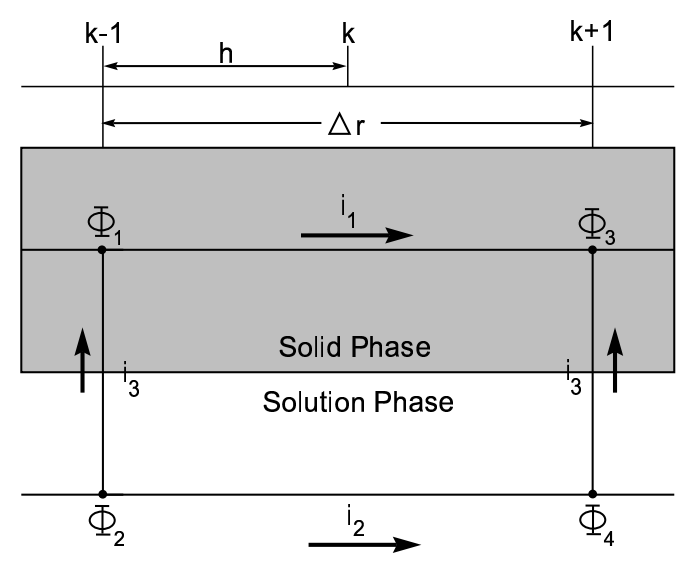

Figure 1. Schematic diagram of a circuit section overlaying a finite region of porous electrode.

Here $V_{1}, V_{2}(\mathrm{~V}), i_{1}^{k}, i_{2}^{k}(\mathrm{~A}), R_{1}^{k}$ and $R_{2}^{k}(\Omega)$, denote the potential differences between the circuit junctions which correspond to the solid and solution phases of the electrode, the currents in the circuit wires and the effective resistances between the circuit junctions which correspond to the solid and solution phases of the electrode, respectively. The resistances are the circuit equivalent to the ohmic resistance experienced by charge carriers moving in the solid and solution phases of the porous electrode. We note that $R_{1}^{k}$ and $R_{2}^{k}$ are given by

$$
R_{1}^{k}=\frac{\Delta r}{A^{k} \sigma} \quad(k=2,4, \ldots, 2 n)
$$

and

$$
R_{2}^{k}=\frac{\Delta r}{A^{k} \kappa} \quad(k=2,4, \ldots, 2 n) .
$$

Since this is a model of a cylindrical electrode, the resistances are functions of the radial co-ordinate and are scaled by the cross-sectional area at the point mid-way between the circuit junctions.

Consider now equation (4), the left-hand-side of which represents the transfer current per unit volume that crosses the solid-solution interface at point $k+1$ in the porous electrode. Given the potentials $\Phi_{3}^{k+1}$ and $\Phi_{4}^{k+1}$ 
in the equivalent circuit section, equation (4) may be rewritten as

$$
i_{2}^{k+2}-i_{2}^{k}=\frac{\Delta r A^{k+1} a i_{0}\left(\alpha_{a}+\alpha_{c}\right) F}{R T}\left(\Phi_{3}^{k+1}-\Phi_{4}^{k+1}\right) \quad(k=2,4, \ldots, 2 n)
$$

so that

$$
i_{3}^{k+1}=\frac{V_{3}}{R_{3}^{k+1}} .
$$

Here $i_{3}^{k+1}(\mathrm{~A})$ denotes the current in the region of the circuit corresponding to the solid-solution interface in the electrode. The potential difference between the circuit junctions, which represents the surface overpotential in the electrode, is denoted by $V_{3}(\mathrm{~V})$. The resistance between the circuit junctions, corresponding to the kinetic resistance experienced by charge as it passes from the solid phase to the solution phase in the electrode, is denoted by $R_{3}^{k+1}(\Omega)$. This resistance is given by

$$
R_{3}^{k+1}=\frac{R T}{\Delta r A^{k+1} a i_{0}\left(\alpha_{a}+\alpha_{c}\right) F} \quad(k=0,2,4, \ldots, 2 n)
$$

where $R_{3}^{1}$ (i.e. $k=0$ ) is obtained by using a second order forward difference approximation in equation (2). Like $R_{1}^{k}$ and $R_{2}^{k}$, the resistance $R_{3}^{k+1}$ is also scaled by the cross-sectional area of the electrode to account for the cylindrical geometry.

The resistances $R_{1}^{k}, R_{2}^{k}$ and $R_{3}^{k+1}$ define the elements of the circuit section shown in Figure 2. This figure shows the equivalent circuit corresponding to a region of porous electrode of width $\Delta r$, under the condition of linear activation polarization.

It follows from above that an equivalent circuit for the entire porous electrode is obtained by combining the equivalent circuit loops for all regions of the electrode.

\section{Solution Technique}

Figure 3 shows the equivalent circuit for a porous cylindrical electrode which has been divided into three finite regions. It is important to note that the circuit contains a number of distinct current loops which cannot be manipulated to form a single current loop. In order to determine the current in each circuit member, Kirchhoff's Rules for a multi-loop circuit may be applied. 


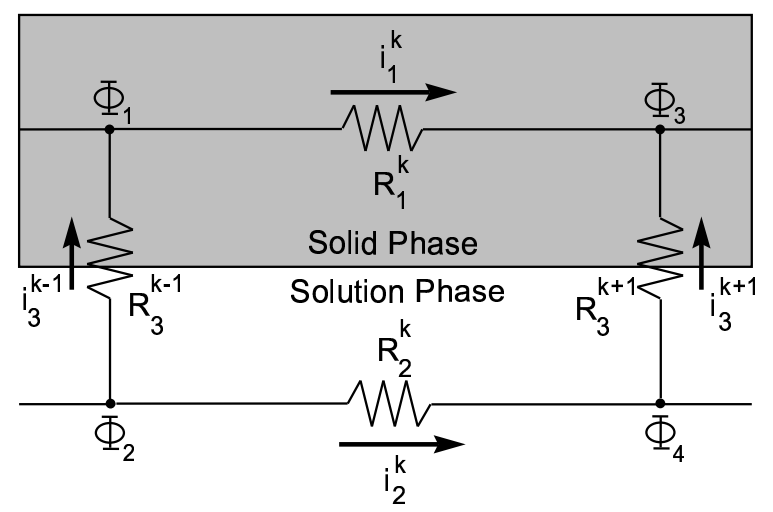

Figure 2. Equivalent circuit section for a finite region of porous electrode.

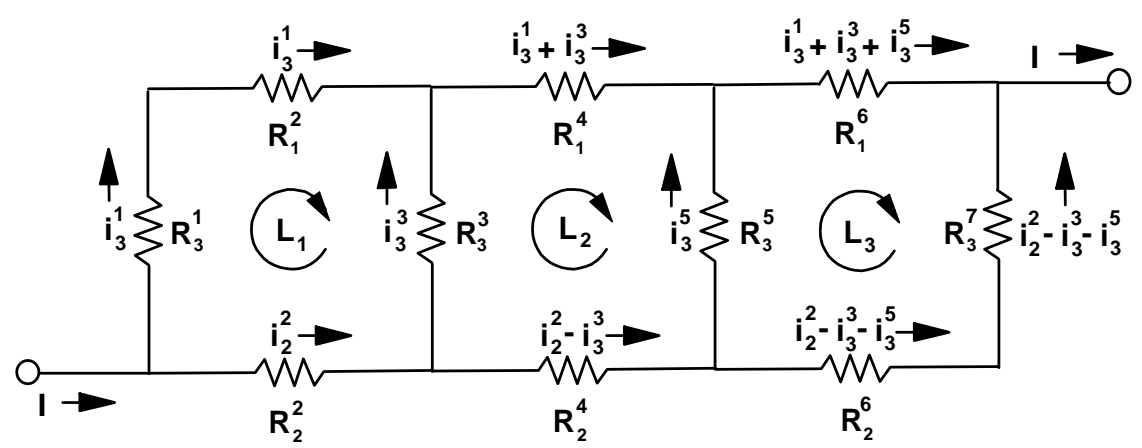

Figure 3. Equivalent circuit for a porous electrode comprised of three finite regions. 
The circuit shown in Figure 3 contains at least three distinct current loops, which are denoted by $L_{1}, L_{2}$ and $L_{3}$. The total applied current, denoted by $I(\mathrm{~A})$, is split into a transfer current, $i_{3}^{1}(\mathrm{~A})$, and a solution phase current, $i_{2}^{2}(\mathrm{~A})$, at the first circuit junction. We define the transfer currents in the second and third vertical members of the circuit to be given by $i_{3}^{3}$ and $i_{3}^{5}$ (A) respectively. Expressions for the currents in each remaining member of the circuit are readily obtained by applying Kirchhoff's First Rule which states that the algebraic sum of the currents entering any node within the circuit is equal to zero. These expressions are shown in the figure.

Traversing each circuit loop in a clockwise direction, as indicated in Figure 3, and applying Kirchhoff's Second Rule, which states that for any closed circuit loop, the algebraic sum of the voltages is equal to zero, leads to a set of linear algebraic equations for the currents $i_{3}^{1}, i_{2}^{2}, i_{3}^{3}$ and $i_{3}^{5}(\mathrm{~A})$ as follows:

$$
\left[\begin{array}{cccc}
1 & 1 & 0 & 0 \\
R_{3}^{1}+R_{1}^{2} & -R_{2}^{2} & -R_{3}^{3} & 0 \\
R_{1}^{4} & -R_{2}^{4} & R_{3}^{3}+R_{1}^{4}+R_{2}^{4} & -R_{3}^{5} \\
R_{1}^{6} & -R_{2}^{6}-R_{3}^{7} & R_{1}^{6}+R_{2}^{6}+R_{3}^{7} & R_{3}^{5}+R_{1}^{6}+R_{2}^{6}+R_{3}^{7}
\end{array}\right]\left[\begin{array}{c}
i_{3}^{1} \\
i_{2}^{2} \\
i_{3}^{3} \\
i_{3}^{5}
\end{array}\right]=\left[\begin{array}{c}
I \\
0 \\
0 \\
0
\end{array}\right]
$$

For a given electrode configuration, expressions (8), (9) and (12) determine the values for the resistances in the above co-efficient matrix. Direct inversion of this matrix yields values for the currents, $i_{3}^{1}, i_{2}^{2}, i_{3}^{3}$ and $i_{3}^{5}(\mathrm{~A})$. From these values, all currents in the circuit may be determined.

The transfer current per unit volume, $j\left(\mathrm{~A} / \mathrm{cm}^{3}\right)$, is a measure of the rate of reaction at the solid-solution interface. We define $j$ to be an anodic current given by

$$
j^{k+1}=\frac{i_{2}^{k+2}-i_{2}^{k}}{\Delta r A^{k+1}}=\frac{-i_{3}^{k+1}}{\Delta r A^{k+1}} \quad(k=0,2,4, \ldots, 2 n) .
$$

Thus, reaction rate values are readily determined from the equivalent circuit results once the transfer currents $i_{3}^{1}, i_{3}^{3}$ and $i_{3}^{5}$ have been calculated.

The above analysis was carried out using 3 finite circuit sections but our results generalize to $n>3$ sections trivially. The results presented here were obtained by running a FORTRAN computer program written to implement the above solution technique for an arbitrary number of electrode sections. Since the equivalent circuit is comprised of a number of "finite circuit loops", we will refer to the process of determining the SCD in this manner as the Finite Difference Equivalent Circuit (FDEC) approach. 


\section{Current Distribution Results}

Figure 4 compares the solution phase current density and reaction distribution results obtained from the FDEC model for various values of the solid and solution phase conductivities, to the corresponding quantities obtained from the exact solution of the model system represented by equations (1) and (2). The exact solution of such a model system is presented by Mak et al. [3]. The physical and kinetic parameters used in the comparison resemble those of a typical EMD D-Cell cathode of the type found in primary alkaline batteries (refer to Table 1 ).

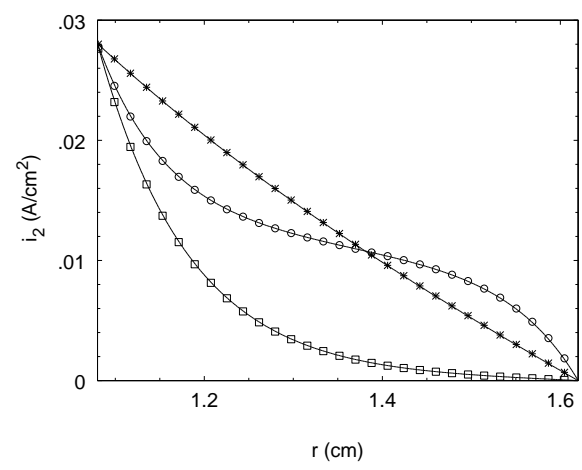

(a)

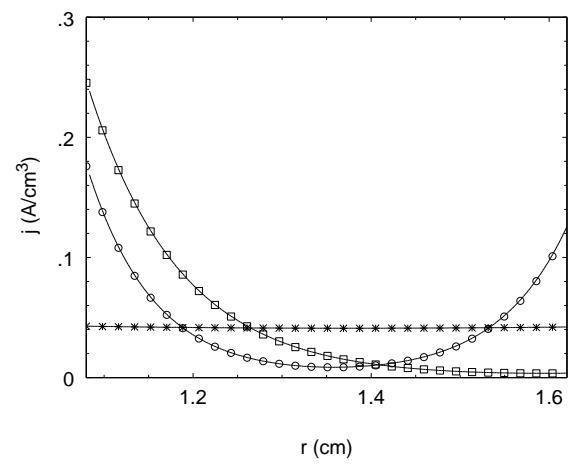

(b)

Figure 4. Comparison of the FDEC solution phase current density results, (a), and reaction distribution results, (b), with the corresponding exact solutions of (1) and (2). : $\sigma=20, \kappa=0.1 ;(\circ): \sigma=0.1, \kappa=0.1 ;(*): \sigma=20, \kappa=20$.

We observe that the distributions obtained by applying the FDEC approach are in very good agreement with the corresponding exact solutions to the model system. In order to illustrate how the equivalent circuit affords an insight into the processes that govern these distributions, we define a dimensionless resistance, $\delta^{k}$. Specifically,

$$
\delta^{k}=\frac{R_{1}^{k}+R_{2}^{k}}{R_{3}^{k+1}} \quad(k=2,4, \ldots, 2 n)
$$

so that

$$
\delta^{k}=\frac{A^{k+1}}{A^{k}} \frac{(\Delta r)^{2} a i_{0} F}{R T}\left[\frac{1}{\sigma}+\frac{1}{\kappa}\right] .
$$


Table 1. Physical and kinetic parameters used for comparing the FDEC approach to the exact solutions.

\begin{tabular}{ll}
\hline \multicolumn{1}{c}{ Parameter } & \multicolumn{1}{c}{ Value } \\
\hline Equivalent circuit node points, $N$ & 300 \\
Discharge rate, & $15(\mathrm{~mA} / \mathrm{g})$ \\
Inner radius of annular cathode, $r_{i}$ & $1.08(\mathrm{~cm})$ \\
Outer radius of annular cathode, $r_{o}$ & $1.62(\mathrm{~cm})$ \\
Height of annular cathode, $H$ & $4.72(\mathrm{~cm})$ \\
Mass of EMD used in the cathode, & $60(\mathrm{~g})$ \\
Pore wall surface area per unit volume [4], a & $1.1 \times 10^{6}(/ \mathrm{cm})$ \\
Exchange current density [5], $i_{0}$ & $2 \times 10^{-7}\left(\mathrm{~A} / \mathrm{cm}^{2}\right)$ \\
\hline
\end{tabular}

The $\delta^{k}$ parameter is the discrete analogue of the dimensionless exchange current parameter initially proposed by Newman and Tobias [6]. This parameter determines the non-uniformity of the reaction distribution within a porous electrode in the absence of concentration effects. Using the FDEC approach we clearly see the physical interpretation of the $\delta^{k}$ parameter. It represents the ratio of ohmic resistance to kinetic resistance at a given point in the electrode.

It is convenient to interpret the ratio $A^{k+1} / A^{k}$ in expression (16), in terms of a discretization error which occurs when we apply the FDEC approach. This error is of order $\Delta r / 2 r^{k}$, and arises because, within the circuit, the ohmic and charge transfer resistances are offset from one another by a distance $\Delta r / 2$. Minimizing this error ensures that the ratio $A^{k+1} / A^{k}$, approaches unity for all possible electrode configurations. This will occur if $\Delta r$ is much smaller than the inner radius of the annular electrode. Setting the number of node points, $N$, in the equivalent circuit to be large, say $N \geq 50$ for standard size cylindrical cells, guarantees that the ratio, $A^{k+1} / A^{k}$ approaches unity. Under these conditions $\delta^{k}$ is independent of $k$ and we denote this limiting value by $\delta$.

When $\delta$ is large the resistances $R_{1}^{k}$ and/or $R_{2}^{k}$ govern the distribution of current in the equivalent circuit. This corresponds to the relative conductivities of the solid and solution phases governing the distribution of current in the porous cathode. In Figure 4 we observe two limiting distribution profiles for this case. Firstly, when $\kappa \ll \sigma\left(\right.$ i.e. $\left.R_{1}^{k} \ll R_{2}^{k}\right)$ the 
comparatively low solution phase conductivity severely limits the length of the current path in the electrolyte solution. In order to minimize the ohmic potential drop in the solution phase the majority of charge crosses the solid-solution interface in a narrow region close to the inner radius of the cathode.

The second type of distribution observed in Figure 4, for $\delta$ large, occurs when $\sigma$ is equal to $\kappa$ and they are both small (i.e. $R_{1}^{k}$ and $R_{2}^{k}$ are equal and large). In this case the majority of cathodic reaction is confined to narrow zones at the inner and outer radii of the cathode. Little reaction occurs in the region mid-way between these two interfaces. The FDEC approach provides us with a good physical interpretation of this distribution. The fact that we observe such a current minimum in the equivalent circuit means that there is a point at which the combined resistive effects of $R_{1}^{k}$ and $R_{2}^{k}$ reach a maximum. The observed current minimum in the equivalent circuit corresponds to a maximum in the combined ohmic resistance of the solid and solution phases of the porous cathode.

When $\delta$ is small the large values of the resistances, $R_{3}^{k+1}$, govern the distribution of current in the equivalent circuit. This corresponds to slow charge transfer kinetics governing the distribution of current in the porous cathode. Charge in the solution phase is distributed uniformly across the width of the cathode. As can be seen in Figure 4, for the case $\sigma=20$, $\kappa=20$, this yields a linear distribution of solution phase current density and a uniform reaction distribution across the cathode.

\section{Curvature Effects}

The cross-sectional area of an annular electrode increases linearly from the inner radius to the outer radius. It is this increase in area, which does not occur for planar electrodes, that characterizes the effect of curvature on the polarization of such electrodes. To illustrate this we begin by defining a dimensionless curvature parameter, $\omega$, given by

$$
\omega=\frac{\mu}{\mu+r^{1}} \quad(0<\omega<1),
$$

where $\mu(\mathrm{cm})$, is the thickness of the electrode and $r^{1}(\mathrm{~cm})$, is the inner radius of the annular electrode. The $\omega$ parameter may be thought of as a measure of the deviation of the surface of the annular electrode from planarity. As $\omega \rightarrow 0$, the electrode configuration is more planar, whereas 
as $\omega \rightarrow 1$, the electrode surface becomes highly curved.

The cross-sectional area, in terms of $\omega$, at any radius within an annular electrode is given by

$$
A^{k}=A^{1}\left\{1+\frac{k-1}{2 n} \frac{\omega}{1-\omega}\right\} \quad(k=1,2, \ldots, 2 n+1) .
$$

Dividing both sides of expression (18) by $A^{1}$ and defining a dimensionless radial length, $k^{*}=(k-1) / 2 n$, we obtain an expression for the crosssectional area of an annular electrode relative to that of a planar electrode having a cross-sectional area equal to that of the annular electrode at the inner radius. Specifically,

$$
A^{*}=\frac{A^{k}}{A^{1}}=1+k^{*}\left(\frac{\omega}{1-\omega}\right) \quad\left(0 \leq k^{*} \leq 1\right) .
$$

Figure 5 shows the behaviour of $A^{*}$ as $k^{*}$ and $\omega$ vary between their limiting values.

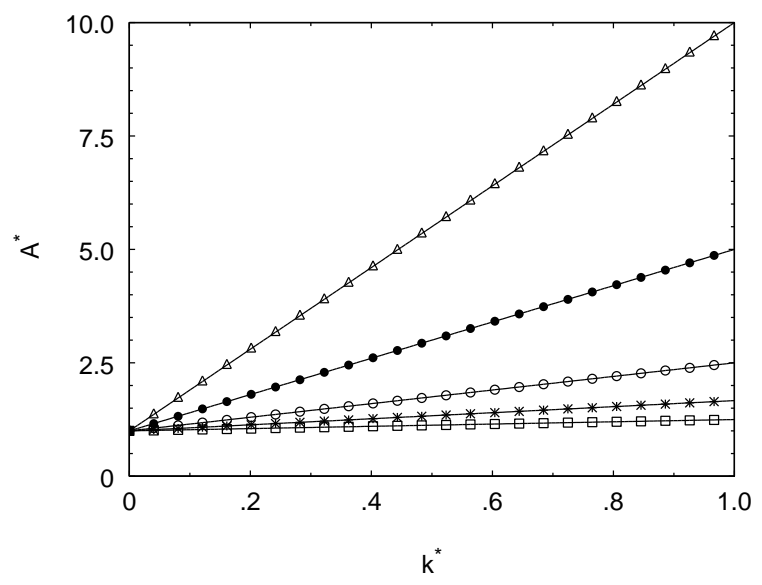

Figure 5. The effect of electrode curvature and dimensionless radial distance on the relative increase in cross-sectional area. : $\omega=0.0 ;(*): \omega=0.2 ;(\circ): \omega=0.6 ;(\bullet)$ : $\omega=0.8 ;(\triangle): \omega=0.9$.

We observe that for non-planar electrodes (i.e. $\omega \neq 0$ ) $A^{*}$ increases linearly as the electrode is traversed from the inner to the outer radius. In addition, we see that the rate of this increase is larger for electrodes having a high curvature. It is this increase in relative area and its dependence on 
the curvature of the electrode that characterizes the curvature effect. The degree to which the curvature effect is evident in the polarization behaviour of an annular electrode depends on the distribution of current within the electrode. We interpret this behaviour by applying the equivalent circuit analogy to the porous annular cathode.

The total potential loss $\Phi_{C}(\mathrm{~V})$, across the cathode, is given by the difference in the solution phase potential at the inner radius and the solid phase potential at the outer radius, of the cathode. In terms of an equivalent circuit, $\Phi_{C}$, may be calculated by choosing any current path between the circuit terminals and summing the potential differences across each resistance as this path is traversed.

In order to calculate $\Phi_{C}$ for different cathode configurations of varying curvature, we substitute expression (18) into the expressions (8), (9) and (12) when determining the resistances within the equivalent circuit. Once these resistance values have been determined for a given $\omega$, the equivalent circuit problem is solved to obtain the currents in each circuit member. The potential loss, $\Phi_{C}$, may then be calculated and the process repeated for a different $\omega$ value.

As a reference to the polarization behaviour within an annular cathode we calculate the corresponding total potential loss, $\Phi_{L}(\mathrm{~V})$, across a planar cathode (i.e. $\omega=0$ ) having a cross-sectional surface area equal to that of the annular cathode at the inner radius. A dimensionless total potential loss, $\Phi^{*}$ may then be defined as

$$
\Phi^{*}=\frac{\Phi_{C}}{\Phi_{L}} .
$$

A plot of $\Phi^{*}$ versus $\omega$, for various values of $\delta$ is shown in Figure 6. We observe that as the curvature of the electrode increases, $\Phi^{*}$ decreases. In general, increasing the curvature causes the degree of polarization of the porous cathode to decrease. However, we note that magnitude of this polarization decrease is dependent on the current distribution within the electrode.

We recall, from Figure 4(b), that when $\sigma \gg \kappa$, the electrode reaction is limited to a narrow zone at the inner radius of the annular cathode. From Figure 6 we observe that, except for extremely high values of the curvature, under these conditions the reaction does not penetrate the annular cathode enough for curvature to have any significant effect on the polarization. 


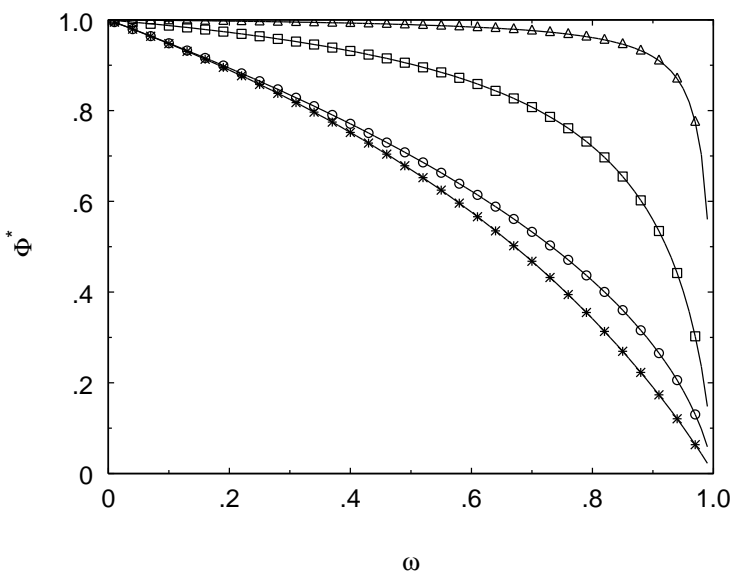

Figure 6. The effect of $\omega$ on $\Phi^{*} .(\triangle): \sigma=20, \kappa=0.001, \delta=0.2 \times 10^{-1} ;: \sigma=20$, $\kappa=0.1, \delta=0.2 \times 10^{-3} ;(\circ): \sigma=0.1, \kappa=0.1, \delta=0.5 \times 10^{-3} ;(*): \sigma=20, \kappa=20$, $\delta=0.2 \times 10^{-5}$.

Comparing Figures 4(b) and 6, we observe that as the reaction rate at the inner radius of the cathode decreases the curvature effect becomes more significant. The most significant reduction in polarization occurs when $\sigma$ equals $\kappa$ and they are both small. Under these conditions the reaction is uniformly distributed across the width of the cathode and the polarization is inversely proportional to the cross-sectional area of the cathode.

\section{Conclusion}

The FDEC approach utilizes an electrical circuit to determine the SCD within annular porous electrodes. The FDEC method provides current and reaction distributions which are consistent with those predicted from conventional mathematical models. The method may also be utilized to analyse the effect of electrode curvature on the polarization behaviour of the electrode.

The FDEC approach to SCD modelling provides an inherent physical interpretation of the SCD results and may be viewed as an interpretive link between the mathematical model and the physical system under consideration. 


\section{Acknowledgments}

The authors wish to acknowledge the support of Delta EMD Australia Pty. Ltd. and the Duracell European Technical Centre during the course of this research.

\section{References}

1. J. Newman and W. Tiedemann, 'Porous-Electrode Theory with Battery Applications', AIChE Journal, 179:25-41, 1975.

2. S. L. Marshall, 'Mathematical Modelling of Interfacial Current Distribution in Cylindrical Porous electrodes', J. Electrochem. Soc., 138:1040-1047, 1991.

3. C. Y. Mak, H. Y. Cheh, G. S. Kelsey and P. Chalilpoyil, 'Modelling of Cylindrical Alkaline Cells: II. Secondary current Distribution', J. Electrochem. Soc., 138:1611-1615, 1991.

4. W. J. Wruck, The Characterization and Modeling of the Alkaline $\mathrm{MnO}_{2}$ Cathode, Ph.D. Thesis, The University of Wisconsin, USA, 1984.

5. J-S. Chen and H. Y. Cheh, 'Modelling of Cylindrical Alkaline Cells: III. Mixed Reaction Model for the Anode', J. Electrochem. Soc., 140:1205-1213, 1993.

6. J. S. Newman and C. W. Tobias, 'Theoretical Analysis of Current Distribution in Porous Electrodes', J. Electrochem. Soc., 109:1183-1191, 1962. 


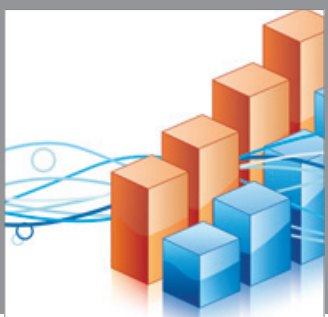

Advances in

Operations Research

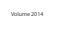

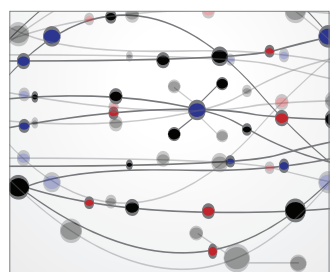

\section{The Scientific} World Journal
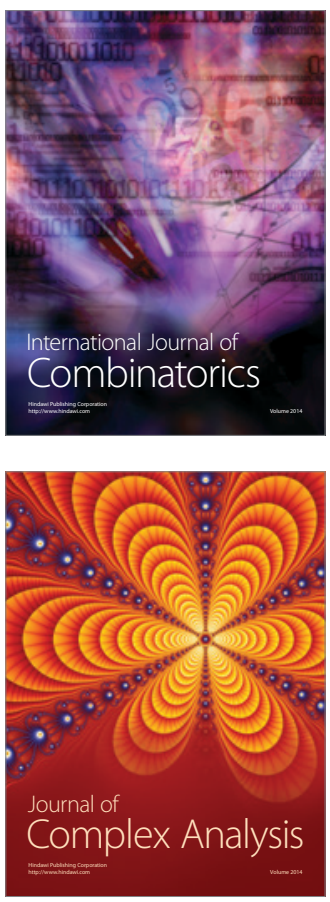

International Journal of

Mathematics and

Mathematical

Sciences
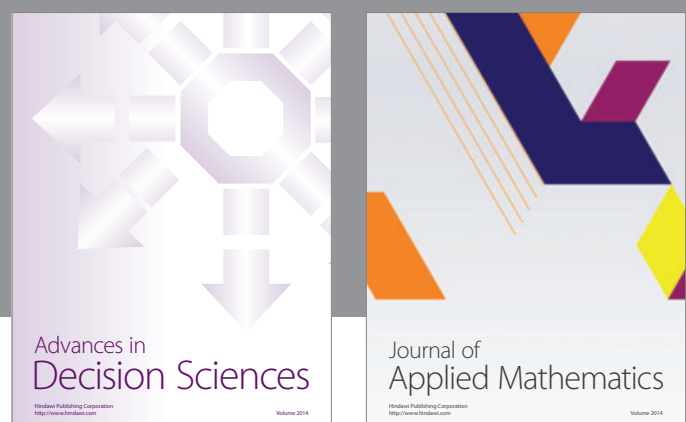

Journal of

Applied Mathematics
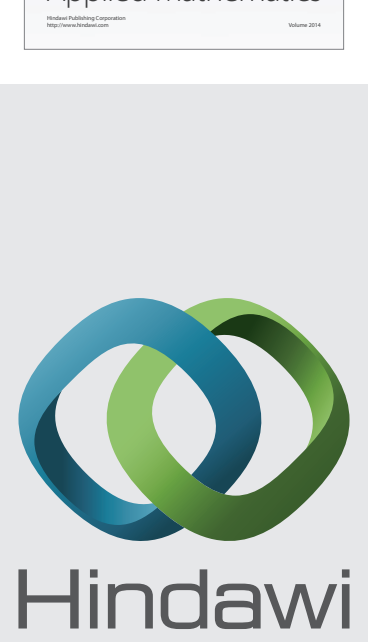

Submit your manuscripts at http://www.hindawi.com
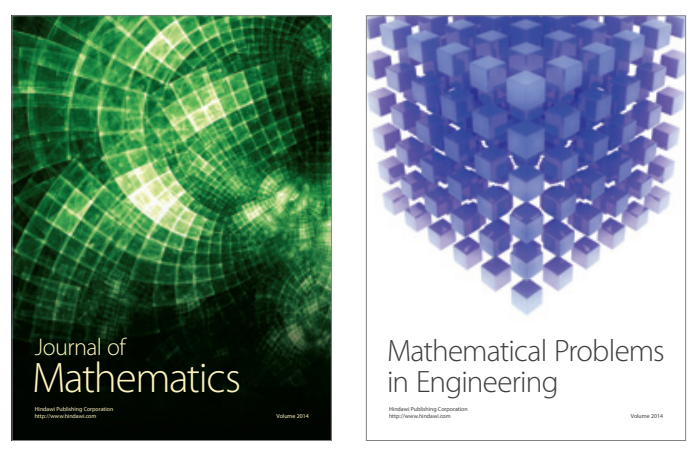

Mathematical Problems in Engineering
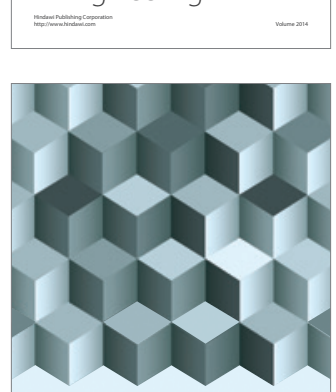

Journal of

Function Spaces
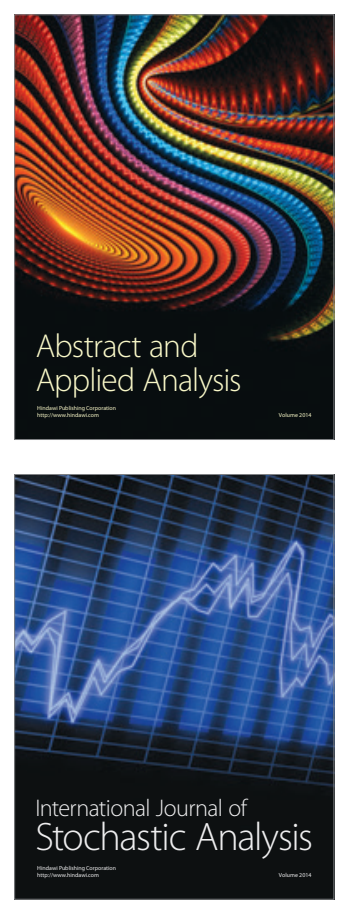

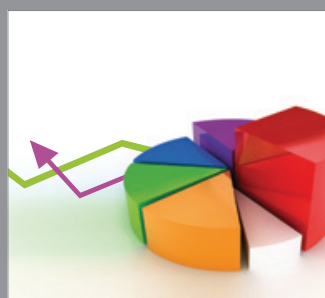

ournal of

Probability and Statistics

Promensencen
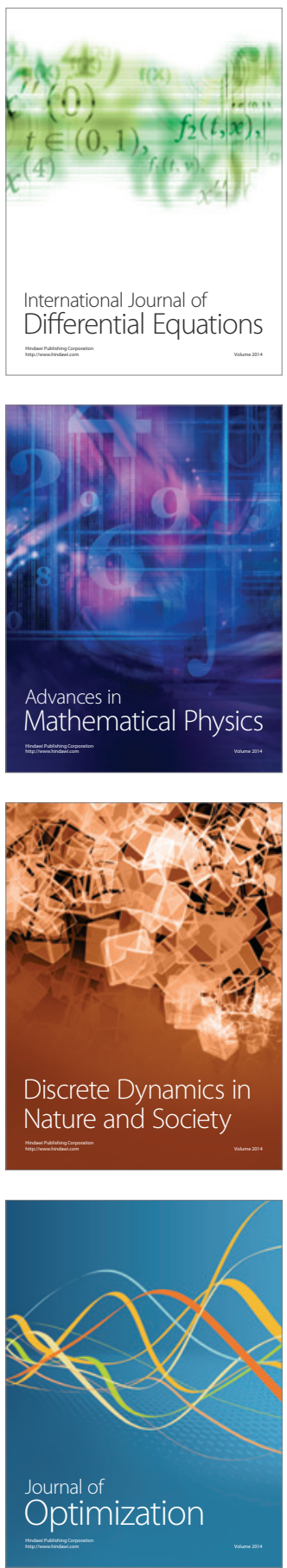